\title{
Modulating substrate choice: the SspB adaptor delivers a regulator of the extracytoplasmic-stress response to the AAA+ protease ClpXP for degradation
}

\author{
Julia M. Flynn, ${ }^{1}$ Igor Levchenko, ${ }^{1,2}$ Robert T. Sauer, ${ }^{1}$ and Tania A. Baker ${ }^{1,2,3}$ \\ ${ }^{1}$ Department of Biology and ${ }^{2}$ Howard Hughes Medical Institute, Massachusetts Institute of Technology, Cambridge, \\ Massachusetts 02139, USA
}

\begin{abstract}
Adaptor proteins help proteases modulate substrate choice, ensuring that appropriate proteins are degraded at the proper time and place. SspB is an adaptor that delivers ssrA-tagged proteins to the AAA+ protease ClpXP for degradation. To identify new SspB-regulated substrates, we examined proteins captured by $\mathrm{ClpXP}^{\text {trap }}$ in $s s p B^{+}$but not $s s p B^{-}$strains. Rse $A^{1-108}$, a fragment of a transmembrane protein that regulates the extracytoplasmic-stress response, fits this criterion. In response to stress, RseA is cleaved on each side of the membrane and is released as a cytoplasmic fragment that remains bound in an inhibitory complex with the $\sigma^{\mathrm{E}}$ transcription factor. Trapping experiments together with biochemical studies show that ClpXP functions in concert with SspB to efficiently recognize and degrade $\operatorname{Rse}^{1-108}$, and thereby releases $\sigma^{\mathrm{E}}$. Genetic studies confirm that $\mathrm{ClpX}$ and $\mathrm{SspB}$ participate in induction of the $\sigma^{\mathrm{E}}$ regulon in vivo, acting at the final step of an activating proteolytic cascade. Surprisingly, the SspB-recognition sequence in $\operatorname{RseA}^{1-108}$ is unrelated to its binding sequence in the ssrA tag. Thus, these experiments elucidate the final steps in induction of the extracytoplasmic stress response and reveal that $\mathrm{SspB}$ delivers a broader spectrum of substrates to ClpXP than has been recognized.
\end{abstract}

[Keywords: RseA; $\sigma^{\mathrm{E}}$; ClpX; ClpP; RpoE]

Received July 14, 2004; revised version accepted August 3, 2004.

The AAA+ protease ClpXP performs a diverse array of cellular tasks, including degrading incomplete polypeptides, adjusting the activity of metabolic enzymes, and altering the levels of regulatory proteins in response to stress (Gottesman et al. 1998; Wang et al. 1999; Maurizi and Rasulova 2002; Flynn et al. 2003; Gottesman 2003). As a result, many substrates compete for degradation by a relatively small number of ClpXP protease molecules (Ortega et al. 2004). The priority of substrate recognition and degradation can also be controlled by adaptor proteins, which enhance or inhibit interactions between specific substrates and ClpXP or other AAA+ proteases (Dougan et al. 2002a). How widely adaptor proteins are used to control substrate choice is not currently understood.

In the ClpXP protease, ClpX-a hexameric-ring ATPase-binds native substrate proteins, denatures these molecules, and translocates the unfolded polypeptides into an internal degradation chamber of the ClpP

${ }^{3}$ Corresponding author.

E-MAIL tabaker@mit.edu; FAX (617) 252-1852.

Article and publication are at http://www.genesdev.org/cgi/doi/10.1101/ gad. 1240104 peptidase (Maurizi et al. 1990, 1994; Wojtkowiak et al. 1993; Wang et al. 1997; Weber-Ban et al. 1999; Kim et al. 2000; Kim and Kim 2003). ClpX binds to short unstructured peptides called recognition signals or degradation tags, usually located near the amino or C terminus of substrates (Levchenko et al. 1997; Gottesman et al. 1998; Gonciarz-Swiatek et al. 1999; Flynn et al. 2003). The ssrA degradation tag is a well-characterized 11-residue peptide (AANDENYALAA), which is added cotranslationally to nascent polypeptides when ribosomes stall (Keiler et al. 1996). SsrA tagging frees these distressed ribosomes for new rounds of translation and targets the incomplete polypeptides for degradation by ClpXP and other proteases (Gottesman et al. 1998; Withey and Friedman 2003).

The SspB adaptor was originally identified by its ability to enhance ClpXP degradation of ssrA-tagged proteins (Levchenko et al. 2000) and is one of the best-characterized proteins that functions in substrate delivery (Wah et al. 2002, 2003; Dougan et al. 2003; Levchenko et al. 2003; Song and Eck 2003; Bolon et al. 2004). SspB enhances recognition of ssrA-tagged proteins by mediating the assembly of ternary complexes in which the substrate, adaptor, and protease are tethered by the following three 
sets of protein-peptide interactions: (1) the AAA+ domain of ClpX binds to the C-terminal $L A A$ sequence of the ssrA tag; (2) the substrate-binding domain of SspB interacts with a sequence spanning the $\mathrm{N}$-terminal seven residues of the ssrA tag; and (3) a short peptide sequence at the end of a flexible SspB tail binds directly to the N-terminal domain of ClpX (Levchenko et al. 2000, 2003; Flynn et al. 2001; Wah et al. 2003; Bolon et al. 2004). Whether SspB delivers any substrates without ssrA tags for ClpXP degradation has not been addressed.

Here, we show that SspB directs ClpXP recognition of Escherichia coli proteins, which are not ssrA tagged. One of these substrates, RseA, functions as a master regulator of the extracytoplasmic-stress response by inhibiting the transcription factor $\left(\sigma^{\mathrm{E}}\right)$ that activates expression of stress genes (De Las Penas et al. 1997b; Missiakas et al. 1997; Dartigalongue et al. 2001; Rezuchova et al. 2003). RseA is a transmembrane protein with an $\mathrm{N}$-terminal cytoplasmic domain, which normally binds to and inhibits $\sigma^{\mathrm{E}}$ (De Las Penas et al. 1997b; Missiakas et al. 1997). In response to the stress-induced accumulation of unfolded or unassembled outer-membrane proteins in the periplasm, RseA is processed via multiple cleavage events in a sequential cascade. DegS protease initially cleaves RseA within its periplasmic domain, activating a second cleavage on the cytoplasmic side of the membrane by YaeL protease (Alba et al. 2001, 2002; Kanehara et al. 2002). These cleavage events release the cytoplasmic domain of RseA from the membrane, but this inhibitory domain remains bound to $\sigma^{\mathrm{E}}$, and thus, additional steps are required before $\sigma^{\mathrm{E}}$ can activate gene expression (Missiakas et al. 1997; Campbell et al. 2003).

Our experiments demonstrate that ClpXP and SspB play a role in the final step of the proteolytic cascade that activates $\sigma^{\mathrm{E}}$. Cleavage of RseA on the cytoplasmic side of the membrane generates a fragment ending in a ClpX recognition signal, similar to the $L A A$ sequence at the end of the ssrA tag. By binding simultaneously to this RseA $^{1-108}$ fragment and ClpX, SspB brings the $\sigma^{\mathrm{E}}$. Rse $\mathrm{A}^{1-108}$ complex and the ClpXP protease together. The RseA fragment is, however, the only component of this complex that is degraded. Surprisingly, the peptide sequences bound by $\mathrm{SspB}$ in $\mathrm{RseA}^{1-108}$ and the ssrA tag are not similar, suggesting the SspB has different modes of protein recognition. These results establish that the SspB adaptor recognizes and delivers different classes of cellular proteins for degradation by ClpXP.

\section{Results}

$S s p B$ influences recognition of a set of ClpXP substrates in vivo

To investigate whether SspB controls ClpXP degradation of proteins without ssrA tags, we compared intracellular substrates captured in an inactive variant of ClpP $\left(\mathrm{ClpP}^{\text {trap}}\right)$ in the presence and absence of SspB (Flynn et al. 2003). Trapping strains were $s m p B^{-}$, which inactivates ssrA tagging (Karzai et al. 1999), and $c l p A^{-}$, which removes another ATPase capable of choosing substrates for ClpP. These mutations eliminate trapping of ssrAtagged and ClpAP substrates. Following capture in $s s p B^{+}$ or $s s p B^{-}$strains, ClpXP substrates were visualized by staining following two-dimensional gel electrophoresis (Fig. 1). This experiment revealed that the majority of cellular substrates do not require $\mathrm{SspB}$ to interact with ClpXP. However, a handful of proteins were clearly more abundant in $\mathrm{ClpP}^{\text {trap }}$ when $\mathrm{SspB}$ was present. This differential trapping indicates that $\mathrm{SspB}$ influences the recognition of a subset of ClpXP substrates. Interestingly, a few proteins were more efficiently trapped when SspB was absent, suggesting that SspB may also inhibit ClpXP degradation of certain proteins.

\section{One SspB-dependent substrate is an N-terminal fragment of RseA}

Tandem-mass spectrometry identified one of the most prominent SspB-dependent ClpXP trapped proteins as an $\mathrm{N}$-terminal fragment of RseA. Tryptic digestion of the RseA spot followed by mass spectrometry identified peptides covering the $\mathrm{N}$-terminal 108 amino acids of RseA (Fig. 2), including a peptide with a molecular weight corresponding to residues 94-108: VRPWAAQLTQMG $V A A^{108}$. The fact that this peptide $\operatorname{did}$ not terminate with lysine or arginine (as expected for an internal tryptic fragment) indicated that alanine was the natural $\mathrm{C}$ terminus of the trapped protein. Thus, this analysis demonstrates that the trapped RseA fragment $\left(\mathrm{RseA}^{1-108}\right)$ terminates with the sequence $V A A-C O O H$ (Fig. 2). This $\mathrm{C}$-terminal sequence is a member of the well-characterized C-motif 1 class of ClpX recognition signals (Flynn et
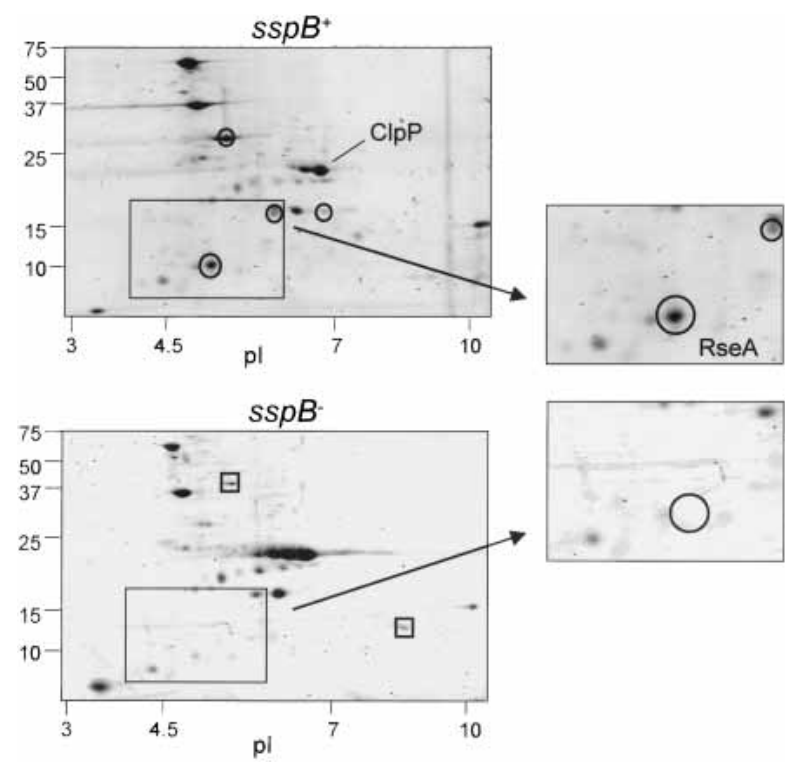

Figure 1. Proteins captured by $C l p X P^{\text {trap }}$ with and without SspB. Two-dimensional gel analysis of proteins captured by ClpXP trap in E. coli strains JF162 (ssp $B^{+} c l p A^{-}$; top) and JF259 (ssp $B^{-} \operatorname{clp} A^{-}$; bottom). Representative proteins trapped preferentially in the $s s p B^{+}$strain are circled, whereas proteins trapped preferentially in the $s s p B^{-}$strain are marked by squares. 


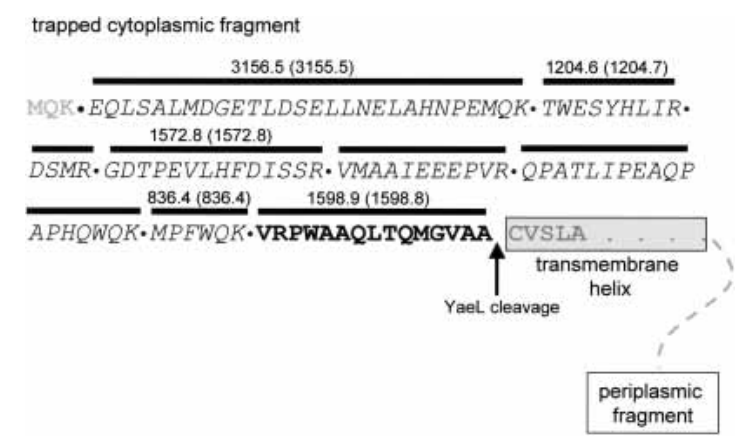

Figure 2. Sequence analysis of the ClpXP $\mathrm{P}^{\text {trap }}$-captured RseA fragment. Tryptic fragments of the RseA fragment were analyzed by mass spectrometry. Identified peptides are marked with bold lines above the corresponding sequences; sequences identified by tandem mass spectrometry are italicized, and peptides identified by MALDI mass spectrometry have the experimental (expected) molecular weights listed. The peptide highlighted in bold was identified by MALDI mass spectrometry and is the C-terminal tryptic peptide of the trapped fragment.

al. 2003), and thus, it makes sense that it would target the RseA fragment to ClpXP.

Recognition of RseA by the cytoplasmic protease ClpXP must occur after YaeL cleavage releases the Nterminal fragment from the membrane (Alba et al. 2001; Kanehara et al. 2002). On the basis of the cleavage specificity of the homologous SP2 protease, Alba et al. (2002) proposed that YaeL might cleave RseA between $A^{108}$ and $C^{109}$, to generate the N-terminal fragment that we trapped and characterized.

\section{Rse $A^{1-108}$ is a substrate for $S s p B$ and ClpXP in vitro}

A fragment corresponding to Rse $\mathrm{A}^{1-108}$ was cloned, overexpressed, and purified to investigate its susceptibility to ClpXP degradation in vitro. ClpXP degraded RseA ${ }^{1-108}$ in a reaction that required ATP (Fig. 3A; data not shown). A mutant variant in which the $\mathrm{C}$-terminal sequence was $V D D^{108}$ (RseA-DD ${ }^{1-108}$ ) was also purified and was found to be degraded 25-30 times more slowly than RseA ${ }^{1-108}$ (Fig. 3A, inset). Thus, we conclude that the C-terminal sequence of $\mathrm{RseA}^{1-108}$ is a critical signal that targets this protein for degradation by ClpXP.

As expected from the trapping results, $\mathrm{SspB}$ also stimulated degradation of Rse $\mathrm{A}^{1-108}$ by ClpXP in vitro (Fig. 3). SspB reduced the Michaelis constant $\left(K_{\mathrm{m}}\right)$ for ClpXP degradation of RseA ${ }^{1-108}$ approximately sevenfold from 1.3 to $\sim 0.2 \mu \mathrm{M}$ and stimulated $V_{\max }$ by $\sim 50 \%$ (Fig. $3 \mathrm{~A})$. Thus, $\mathrm{SspB}$ enhances productive interactions between RseA ${ }^{1-108}$ and ClpX, in a manner analogous to its role in delivering ssrA-tagged proteins for ClpXP degradation (Levchenko et al. 2000). A truncated SspB variant lacking the tails that bind ClpX did not enhance ClpXP degradation of RseA ${ }^{1-108}$, demonstrating that tethering interactions between SspB and ClpX are important for delivery of this substrate (data not shown).

YaeL cleavage releases the N-terminal fragment of RseA from the membrane, but does not disrupt its bind- ing to $\sigma^{\mathrm{E}}$ (Alba et al. 2002; Kanehara et al. 2002). We asked, therefore, whether SspB could deliver the $\sigma^{\mathrm{E}} \cdot \mathrm{RseA}^{1-108}$ complex to ClpXP for disassembly and degradation. As shown in Figure 3B, ClpXP degraded RseA ${ }^{1-108}$ bound to $\sigma^{\mathrm{E}}$, and SspB stimulated this degradation. At the concentrations tested, the rate of degradation of free RseA ${ }^{1-108}$ was similar to that of complexed Rse $^{1-108}$. This result indicates that binding of $\sigma^{\mathrm{E}}$ to RseA $^{1-108}$ does not inhibit degradation or provide any critical contacts that enhance recognition of Rse $\mathrm{A}^{1-108}$ by ClpX. Importantly, $\sigma^{\mathrm{E}}$ in the $\sigma^{\mathrm{E}} \cdot \mathrm{RseA}^{1-108}$ complex was not degraded. In addition, as expected, SspB remained undegraded throughout the reaction (data not shown).

On the basis of this analysis, we conclude that SspB
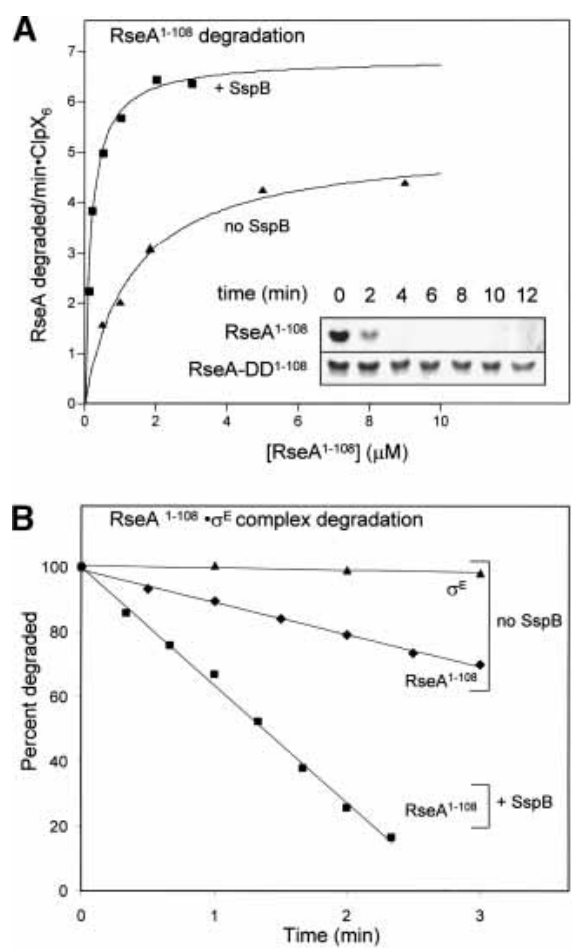

Figure 3. ClpXP efficiently degrades purified RseA ${ }^{1-108}$ in an SspB-stimulated manner. (A) Rates of ClpXP-mediated degradation of ${ }^{35}$ S-labeled RseA ${ }^{1-108}$ by $\mathrm{ClpX}_{6}(50 \mathrm{nM})$ and $\mathrm{ClpP}_{14}$ (150 $\mathrm{nM}$ ) were determined at different substrate concentrations in the presence or absence of SspB (200 nM). Degradation was assayed by changes in TCA-soluble radioactivity, and rates were plotted against the substrate concentration. The solid lines are fits to the Michaelis-Menten equation in the absence $\left(\mathrm{K}_{\mathrm{m}}=1.3\right.$ $\left.\mu \mathrm{M}, \mathrm{V}_{\max }=5.2 \mathrm{~min}^{-1}\right)$ or presence $\left(\mathrm{K}_{\mathrm{m}}=0.18 \mu \mathrm{M}, \mathrm{V}_{\max }=6.8\right.$ $\mathrm{min}^{-1}$ ) of SspB. (Inset) Degradation of RseA-DD ${ }^{1-108}(2 \mu \mathrm{M})$ or $\operatorname{RseA}^{1-108}(2 \mu \mathrm{M})$ by $\mathrm{ClpX}_{6}(300 \mathrm{nM})$ and $\mathrm{ClpP}_{14}(800 \mathrm{nM})$ was assayed by SDS-PAGE. $(B)$ ClpXP degradation of RseA ${ }^{1-108}$ complexed with $\sigma^{\mathrm{E}} \cdot{ }^{35} \mathrm{~S}$-labeled RseA ${ }^{1-108}(500 \mathrm{nM})$ was incubated with unlabeled $\sigma^{\mathrm{E}}(500 \mathrm{nM})$ for $5 \mathrm{~min}$ at $30^{\circ} \mathrm{C}$. Degradation by $\mathrm{ClpX}_{6}(50 \mathrm{nM})$ and $\mathrm{ClpP}_{14}(150 \mathrm{nM})$ was assayed by changes in TCA-soluble radioactivity in the presence $(\square)$ or absence ( $)$ of SspB $(200 \mathrm{nM}) \cdot{ }^{35}$ S-labeled $\sigma^{\mathrm{E}}$ was also incubated with unlabeled RseA $^{1-108}$ and ClpXP degradation was monitored in the same manner $(\mathbf{\Lambda})$. No detectable $\sigma^{\mathrm{E}}$ degradation by ClpXP was observed in the presence of SspB. 
can deliver the $\sigma^{\mathrm{E}} \cdot \mathrm{RseA}^{1-108}$ complex to ClpXP, leading to the targeted degradation of Rse $\mathrm{A}^{1-108}$. These results are integrated into a model for $\sigma^{\mathrm{E}}$ activation shown in Figure 4. Following DegS and YaeL cleavage of RseA, SspB delivers the $\sigma^{\mathrm{E}} \cdot$ RseA $^{1-108}$ complex to ClpX, which selectively denatures $\mathrm{RseA}^{1-108}$ and translocates it into $\mathrm{ClpP}$ for degradation. This processing of the RseA fragment by ClpXP releases SspB and $\sigma^{\mathrm{E}}$ from the enzyme complex. As a consequence, $\sigma^{\mathrm{E}}$ is liberated to bind to core RNA polymerase and activate transcription.

\section{SspB and ClpX enhance activation of the $\sigma^{E}$ regulon} in vivo

Taken together, the results presented so far suggest that degradation mediated by ClpXP and SspB controls the intracellular levels of RseA ${ }^{1-108}$ and should therefore influence $\sigma^{\mathrm{E}}$ activity. To test for roles for ClpX and SspB in the extracytoplasmic stress response, we monitored induction of a $\sigma^{\mathrm{E}}$-controlled lacZ reporter gene following induction of the stress response in $s s p B^{-}$or $c l p X^{-}$cells. Extracytoplasmic stress was induced using a plasmidencoded fusion protein, ending with a YYF sequence, which is targeted to the periplasm and activates DegS degradation of RseA (Walsh et al. 2003). Following induction, $\sigma^{\mathrm{E}}$-dependent $\beta$-galactosidase synthesis was delayed in both the $c l p X^{-}$and $s s p B^{-}$cells (Fig. 5). These data show that ClpX and SspB participate in activation of $\sigma^{\mathrm{E}}$ during the stress response. The $c l p X^{-}$cells had a larger defect than the $s s p B^{-}$cells, in accordance with the observation that SspB is not essential for ClpXP degradation of RseA ${ }^{1-108}$ in vitro. Although clearly reduced, the $\sigma^{\mathrm{E}}$-reporter gene was still induced in the absence of
ClpX, suggesting that proteases in addition to ClpXP also participate in the activation of $\sigma^{\mathrm{E}}$ by degrading $\mathrm{RseA}^{1-108}$ (see Discussion).

SspB forms stable delivery complexes with Rse $A^{1-108}$ and with $\sigma^{E} \cdot$ Rse $A^{1-108}$

Mutagenic and crystallographic studies have identified detailed interactions between the ssrA tag and SspB, and peptide-binding studies have established a strong consensus sequence for $\mathrm{SspB}$ recognition of the tag (Levchenko et al. 2000, 2003; Flynn et al. 2001; Song and Eck 2003). Inspection of the Rse ${ }^{1-108}$ sequence, however, failed to identify any sequences with significant homology to the SspB-recognition sequence in the ssrA tag. Thus, we sought to determine whether SspB forms a specific complex with RseA ${ }^{1-108}$ as it does with the ssrA tag using gel filtration as a binding assay. SspB and RseA $^{1-108}$ coeluted on a Superose 12 column at a position distinct from free RseA ${ }^{1-108}$ (Fig. 6A). Moreover, a larger ternary complex was formed when $\mathrm{SspB}, \sigma^{\mathrm{E}}$, and RseA $^{1-108}$ were mixed (Fig. 6B). The presence of SspB, $\sigma^{\mathrm{E}}$, and $\mathrm{RseA}^{1-108}$ in this complex was confirmed by SDSPAGE (data not shown). Stable formation of this ternary complex provides further support for the model that SspB binds the $\sigma^{\mathrm{E}} \cdot \mathrm{RseA}^{1-108}$ complex and delivers this complex to ClpXP.

Truncation experiments established that a sequence near the $\mathrm{C}$ terminus of $\mathrm{RseA}^{1-108}$ was required for stable complex formation with $\mathrm{SspB}$. A truncated variant ending at residue $89\left(\mathrm{RseA}^{1-89}\right)$ failed to coelute with SspB during gel filtration, whereas a slightly longer variant, $\mathrm{RseA}^{1-99}$, retained the ability to bind SspB stably (Fig.

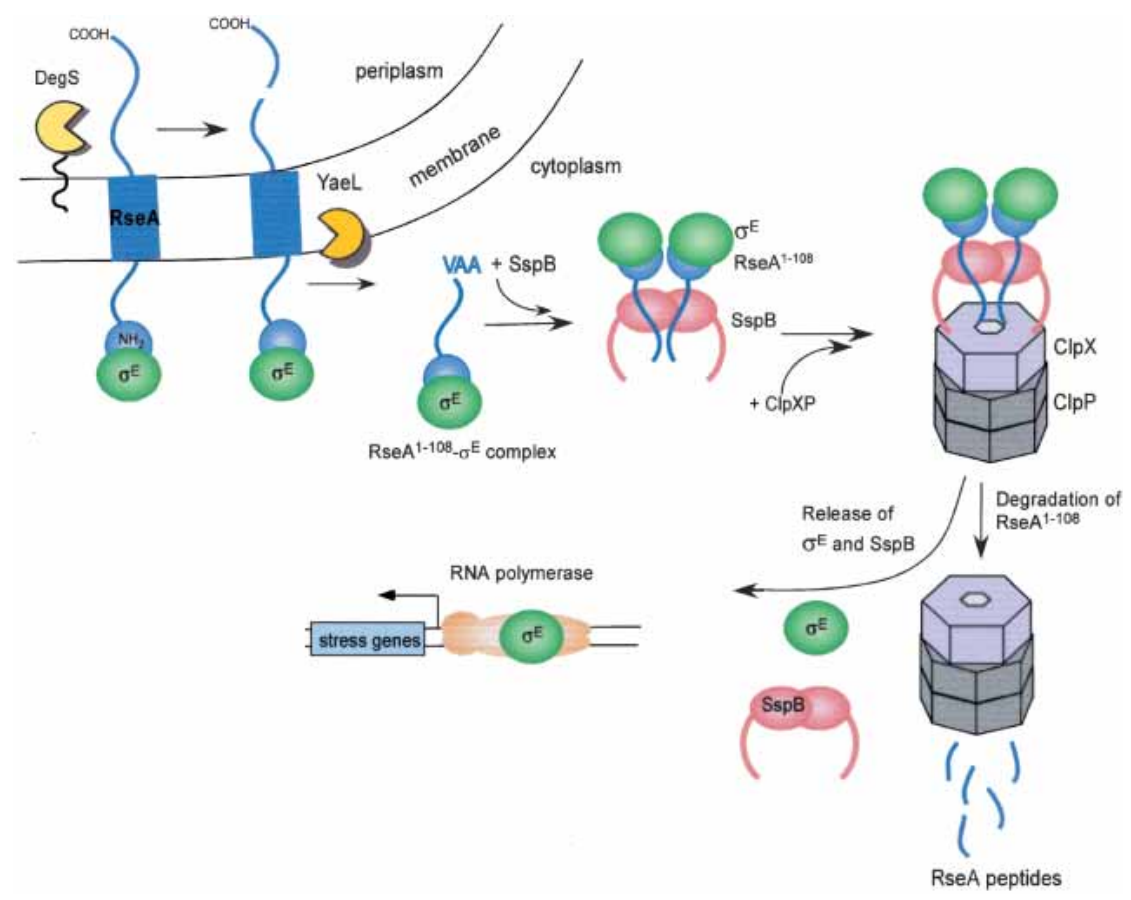

Figure 4. Activation of $\sigma^{\mathrm{E}}$ mediated by a cascade of RseA proteolysis. 
Flynn et al.

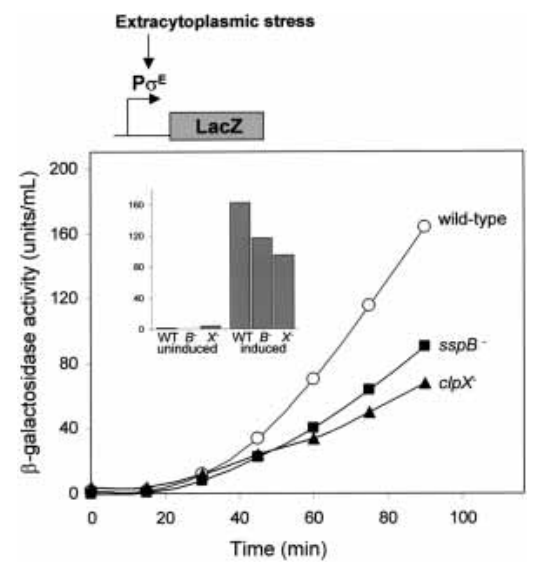

Figure 5. Induction of the $\sigma^{\mathrm{E}}$ regulon is attenuated in $s s p B^{-}$and $c l p X^{-}$strains. The extracytoplasmic-stress response was induced in wild-type (CAG43583), sspB::kan (CAG43583) and clpX::kan (CAG43583) strains with L-arabinose at time 0 . Samples were analyzed for $\beta$-galactosidase activity at the times indicated. The $c l p X::$ kan and $\operatorname{ssp} B::$ kan strains grow slightly slower than wild type. When the cultures were at a similar O.D. ${ }_{600}$, however, the $\operatorname{clpX}::$ kan and $\operatorname{ssp} B::$ kan strains still exhibited reduced levels of $\beta$-galactosidase (see inset). The uninduced samples were measured at time 0 when the cultures were at an O.D. ${ }_{600}$ of 0.15 . The induced samples were measured when the cultures reached an O.D. ${ }_{600}$ of 0.45 .

7A). To determine which portion of RseA ${ }^{1-108}$ bound $\mathrm{SspB}$, we looked for sites protected from tryptic cleavage in the complex. Incubation of RseA ${ }^{1-99}$ with trypsin resulted in two major stable fragments; the larger fragment resulted from digestion after $K^{93}$, whereas the smaller fragment was generated by trypsin digestion after both $R^{58}$ and $K^{93}$ (Fig. 7B). In the presence of $\mathrm{SspB}$, two larger fragments were also observed as a result of partial suppression of the cleavage following $K^{93}$. These data, like the truncation experiments, implicate the sequence surrounding residue 93 in SspB - RseA complex formation.

Peptide-binding studies confirm that the C-terminal region of $\mathrm{RseA}^{1-108}$ mediates its interaction with SspB. A synthetic fluorescein-labeled peptide containing RseA residues $77-108$ bound $\mathrm{SspB}$ with a $K_{d}$ of $0.35 \mu \mathrm{M}$ as determined by changes in fluorescence polarization (Fig. 7C). This binding was competed both by excess RseA $^{1-108}$ and by an ssrA peptide (data not shown). Furthermore, a mutation in the peptide-binding cleft of SspB (Bolon et al. 2004) prevented binding of both molecules. These experiments suggest that the $\mathrm{C}$-terminal region of RseA $^{1-108}$ and the ssrA peptide bind to at least some common sites within the peptide-binding cleft on SspB, despite the lack of significant sequence homology.

\section{Discussion}

ClpXP and $S s p B$ regulate $\sigma^{E}$ activity via RseA destruction

The activity of $\sigma^{\mathrm{E}}$, the transcription factor for the extracytoplasmic-stress response, is tightly controlled by its binding to and inhibition by the transmembrane regulator, RseA (Fig. 4; De Las Penas et al. 1997b; Missiakas et al. 1997). Stress induces sequential cleavages of RseA on each side of the membrane by the DegS and YaeL proteases, respectively, releasing the $\sigma^{\mathrm{E}} \cdot \mathrm{RseA}^{1-108}$ complex into the cytoplasm (Ades 2004). Our results show that $\mathrm{ClpXP}$, with the assistance of $\mathrm{SspB}$, recognizes the inhibited $\sigma^{\mathrm{E}} \cdot \mathrm{RseA}^{1-108}$ complex and catalyzes release of active $\sigma^{\mathrm{E}}$ through selective proteolytic destruction of RseA $^{1-108}$. Thus, SspB and ClpXP participate in the final stage of a proteolytic cascade, which begins in the periplasm and, ultimately, releases an active transcription factor in the cytoplasm.

ClpXP is especially well suited to recognize and degrade proteins with $\mathrm{C}$-terminal signals generated by prior proteolytic cleavage. Cleavage of RseA from the membrane generates a fragment that terminates with $V A A$ $\mathrm{COOH}$, a sequence that belongs to the C-motif 1 class of ClpXP recognition signals (Flynn et al. 2003). For this class of peptide sequences, which includes the ssrA tag, the nonpolar side chains and the free $\alpha$-carboxyl group are both important for ClpX recognition (Kim et al. 2000; Flynn et al. 2001). Thus, a VAA or LAA sequence is recognized poorly, if at all, at an internal position in a protein. Degradation of the SOS response repressor, LexA, also illustrates this type of regulation (Neher et al. 2003a). Full-length LexA is not a ClpXP substrate, but damage-induced auto-cleavage creates an $\mathrm{N}$-terminal LexA fragment, ending with $V A A-C O O H$, which is degraded efficiently by ClpXP (Neher et al. 2003a). Thus, certain internal peptide sequences function as cryptic

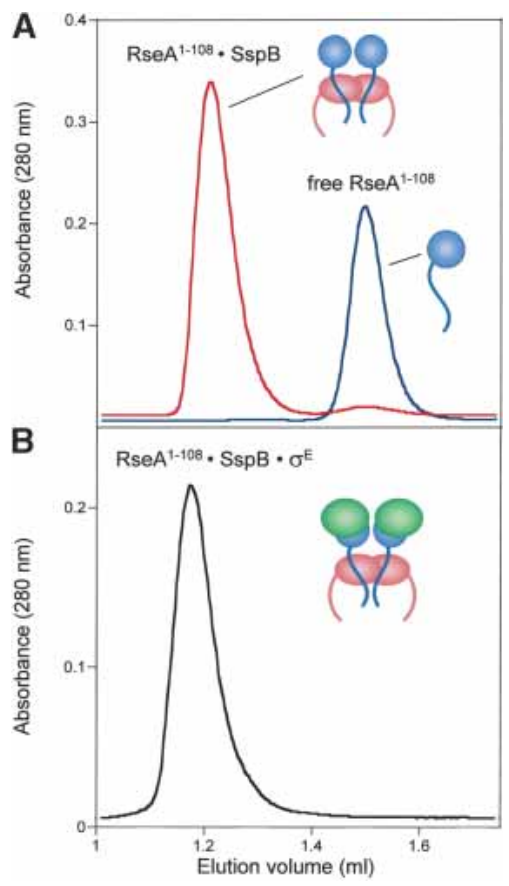

Figure 6. SspB forms stable complexes with $\mathrm{RseA}^{1-108}$ and RseA $^{1-108} \cdot \sigma^{\mathrm{E}}$. Gel-filtration on a Superose 12 column $\left(4^{\circ} \mathrm{C}\right)$ of Rse $^{1-108} \cdot$ SspB complex $(A$, top trace), free RseA $(A$, bottom trace), and $\operatorname{RseA}^{1-108} \cdot \sigma^{\mathrm{E}} \cdot \operatorname{SspB}$ complex $(B)$. 
A
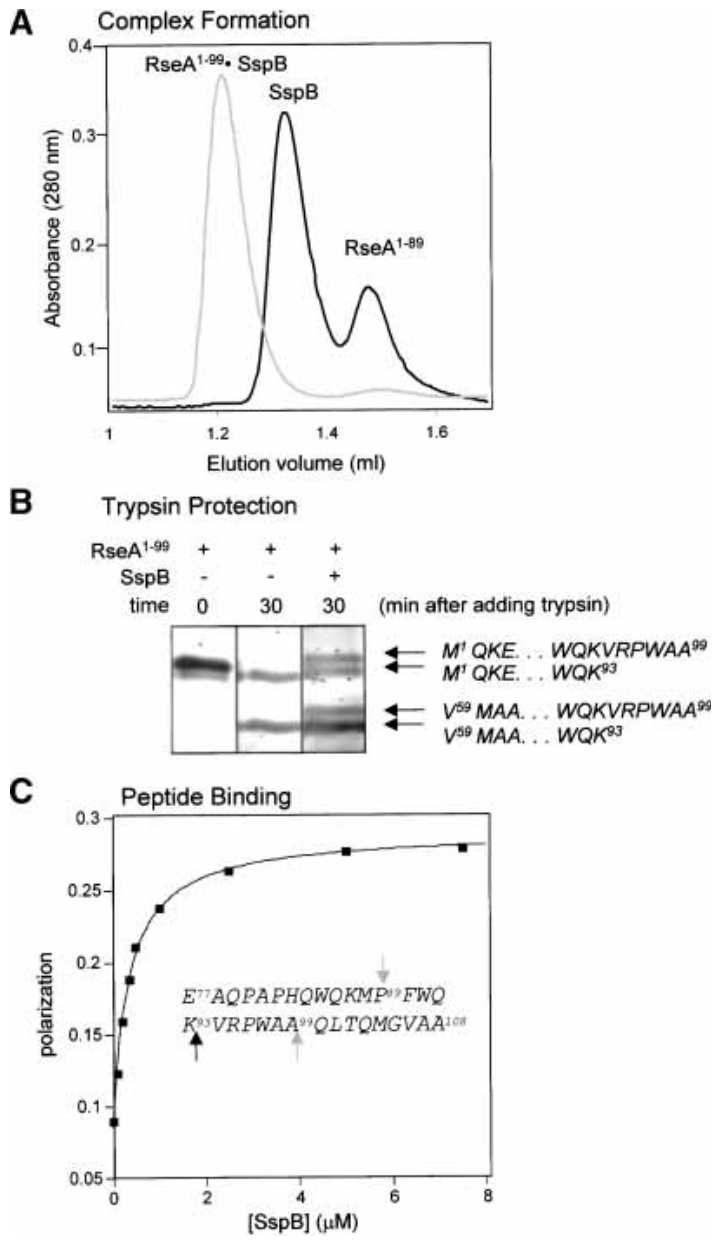

Figure 7. SspB interacts with residues 77-99 of RseA. (A) Rse $^{1-99}$ forms a stable complex with SspB (gray trace), whereas RseA $^{1-89}$ does not form this complex (black trace). The RseA variants and $\mathrm{SspB}$ were incubated at $30^{\circ} \mathrm{C}$ for $5 \mathrm{~min}$ and then chromatographed on a Superose 12 gel-filtration column $\left(4^{\circ} \mathrm{C}\right)$. (B) Protection of $\mathrm{RseA}^{1-99}$ by SspB from tryptic cleavage. RseA $^{1-99}(5 \mu \mathrm{M})$ was incubated with trypsin in the absence or presence of SspB $(15 \mu \mathrm{M})$. Electrospray mass spectrometry and $\mathrm{N}$-terminal sequencing determined the identity of the resulting fragments. $(C)$ The RseA ${ }^{77-108}$ peptide binds to SspB. Binding of fluorescently labeled $\mathrm{RseA}^{77-108}$ peptide to $\mathrm{SspB}$ at $30^{\circ} \mathrm{C}$ was measured by an increase in polarization. The solid line is a fit for a $\mathrm{K}_{\mathrm{d}}$ of $0.35 \mu \mathrm{M}$. Unlabeled RseA ${ }^{77-108}$ peptide was able to compete for binding to the fluoresceinated peptide. The sequence of the RseA ${ }^{77-108}$ peptide is given in the inset. Gray arrows correspond to the C-terminal residues of the fragments tested for complex formation in $A$. The black arrow corresponds to the protected trypsin site in $B$.

degradation signals, which remain hidden until revealed by protein cleavage. Cryptic signals permit coordinated protein destruction, allowing a single protein processing event-such as cleavage in response to an environmental cue-to trigger recognition by ClpXP.

Structural and biochemical studies demonstrate that complexes of $\sigma^{\mathrm{E}}$ with RseA are very stable and incompatible with transcriptional activation. The cocrystal structure of RseA ${ }^{1-90}$ bound to $\sigma^{\mathrm{E}}$ reveals extensive con- tacts in which the first 66 amino acids of RseA are sandwiched between the two domains of $\sigma^{\mathrm{E}}$ in a manner that would directly block $\sigma^{\mathrm{E}}$-RNA polymerase interaction (Campbell et al. 2003). We found that the $\sigma^{\mathrm{E}} \cdot \mathrm{RseA}^{1-108}$ complex copurified over several columns without detectable dissociation during a period of days (J. Flynn and I. Levchenko, unpubl.), and direct experiments estimate the half-life of the complex in vitro to be well in excess of $2 \mathrm{~h}$ (I. Grigorova and C. Gross, pers. comm.). Response to extracytoplasmic stress, in contrast, occurs in minutes, a time-scale similar to the rate of ClpXP degradation of RseA ${ }^{1-108}$ in a $\sigma^{\mathrm{E}} \cdot \mathrm{RseA}^{1-108}$ complex. Therefore, ClpX must actively pull the two proteins in the $\sigma^{\mathrm{E}} \cdot \mathrm{RseA}^{1-108}$ complex apart to release $\sigma^{\mathrm{E}}$ and allow degradation of $\mathrm{RseA}^{1-108}$. The proteolytic activity of $\mathrm{ClpP}$ in the ClpXP complex may assist in activation of $\sigma^{\mathrm{E}}$ by destroying RseA ${ }^{1-108}$ to prevent reformation of the RseA $^{1-108} \cdot \sigma^{\mathrm{E}}$ complex. Thus, a key feature of $\sigma^{\mathrm{E}}$ activation is the mechanical disassembly of the $\sigma^{\mathrm{E}} \cdot \mathrm{RseA}^{1-108}$ complex by ClpXP.

In the cocrystal structure of $\sigma^{\mathrm{E}} \cdot \mathrm{RseA}^{1-90}$, the first 66 residues of RseA form a stable domain that binds $\sigma^{\mathrm{E}}$, whereas the last 24 residues are not visible and are presumably unstructured (Campbell et al. 2003). Although previously there was no known function for this unstructured extension of N-RseA, our data indicates that this region functions to interact with both $\mathrm{SspB}$ and ClpX during the final step of activation of $\sigma^{\mathrm{E}}$. SspB, $\sigma^{\mathrm{E}}$ and N-RseA form a stable delivery complex, in which $\sigma^{\mathrm{E}}$ interacts with the first 66 residues of RseA, and SspB binds to the C-terminal unstructured tail. Why is RseA ${ }^{1-}$ 108 the only member of this stable complex degraded? Both $S \operatorname{spB}$ and $\sigma^{\mathrm{E}}$ probably lack degradation signals that would allow ClpX to engage these proteins to initiate protein degradation. Alternatively, the geometry of the complex might place $\mathrm{RseA}^{1-108}$ but not the other proteins in a position that allows engagement by the enzyme.

$\sigma^{\mathrm{E}}$ function is essential in E. coli (De Las Penas et al. 1997a) but ClpX, ClpP, and SspB are nonessential proteins, suggesting that other proteases also degrade RseA $^{1-108}$ and release active $\sigma^{\mathrm{E}} \cdot \operatorname{clp} X^{-}$and $\operatorname{ssp} B^{-}$cells show reduced induction of a $\sigma^{\mathrm{E}}$-regulated promoter, rather than no induction. In fact, recent experiments demonstrate that several different proteases participate in degradation of $\mathrm{RseA}^{1-108}$, although ClpXP plays the single largest role (R. Chaba and C. Gross, pers. comm.). Hence, RseA ${ }^{1-108}$ must contain targeting signals for several proteases, emphasizing the critical nature of its destruction.

\section{Adaptors like SspB expand and regulate the substrate} repertoire of proteases

Prior to this study, ssrA-tagged proteins were the only known substrate partners for SspB (Levchenko et al. 2000). Identification of RseA ${ }^{1-108}$ as a new SspB partner provides the opportunity to compare mechanisms of substrate delivery. There are many similarities. Both RseA $^{1-108}$ and ssrA-tagged proteins contain a C-motif 1 degradation tag at the extreme $\mathrm{C}$ terminus, and $\mathrm{SspB}$ 
binds to a nearby region within 10-30 residues. For both classes of substrates, SspB enhances ClpXP degradation principally by decreasing $K_{m}$, and therefore serves to stabilize enzyme-substrate interactions. Finally, RseA ${ }^{1-108}$ and the ssrA tag appear to occupy overlapping binding sites in the peptide-binding cleft on SspB.

Despite these similarities, the sequences within Rse $^{1-108}$ and the ssrA tag that bind SspB are not similar. Experiments presented here reveal that the SspB-binding site in RseA ${ }^{1-108}$ lies between residues 77 and 99 (see Fig. 7). This region, as well as the rest of $\mathrm{RseA}^{1-108}$, is devoid of sequences resembling the ssrA tag consensus for SspB binding $\left([\mathrm{AGPSV}]^{1}-[\mathrm{ASV}]^{2}-[\mathrm{NH}]^{3}-[\mathrm{DCE}]^{4}-\mathrm{X}^{5}-\mathrm{X}^{6}-[\mathrm{FWY}]^{7}\right.$; Flynn et al. 2001). Studies are currently in progress to define more clearly how Rse ${ }^{1-108}$ binds to SspB and how the peptide-binding cleft of SspB can interact strongly and specifically with two, seemingly unrelated, sequences.

The studies reported here revealed several different proteins that were trapped in $s s p B^{+}$but not $s s p B^{-}$strains. In addition to $\mathrm{RseA}^{1-108}$, trapping of both AceA (isocitrate lyase) and Cdd (deoxycytidine deaminase) was also stimulated by the presence of SspB (data not shown). Delivery of ssrA-tagged substrates or RseA ${ }^{1-108}$ for ClpXP degradation is clearly a direct consequence of SspB function, and we suspect that additional proteins will also be directly delivered by SspB. However, adaptors also can have indirect effects on substrate selection by AAA+ proteases. For example, by mediating efficient degradation of specific substrates, an adaptor may serve to free the protease to degrade other substrates more efficiently. In addition, targeted degradation of transcription factors, translation regulators, chaperones, and proteases has the potential to cause large changes in protein levels, leading to indirect changes in the repertoire of substrates available for degradation.

Although SspB is a positive regulator of RseA ${ }^{1-108}$ recognition, it also has the potential to act as an inhibitor. In our experiments, ClpXP trapped a few substrates more efficiently when SspB was absent (see Fig. 1). SspB binding could prevent ClpXP degradation of certain proteins by masking their degradation tags. In fact, both SspB and the ClpS adaptor protein inhibit ClpAP recognition of ssrA-tagged proteins (Flynn et al. 2001; Dougan et al. $2002 \mathrm{~b}$ ). Alternatively, absence of competition could lead to improved degradation of substrates or substrate-adaptor complexes that compete with SspB for tethering to ClpX.

It is becoming increasingly clear that many proteins are targeted for disassembly and destruction by AAA+ ATPases both by intrinsic recognition tags and by extrinsic tethering mediated by adaptor proteins. How many adaptors exist for each enzyme, and their overall impact on recognition is not yet known. In addition to $S \mathrm{spB}, E$. coli ClpXP uses the RssB adaptor that delivers the stationary $\sigma$ factor $\sigma^{\mathrm{S}}$ to ClpXP for degradation during nonstarvation conditions (Muffler et al. 1996; Zhou and Gottesman 1998). Furthermore, the UmuD subunit of the UmuD UmuD' heterodimer functions as an SspB-like adaptor for UmuD' degradation by ClpXP during recovery from DNA damage (Neher et al. 2003b).
Why do certain substrates use adaptors? One answer is that adaptor proteins can increase the efficiency of recognition at low substrate concentrations. For example, SspB improves ClpXP recognition of $\mathrm{RseA}^{1-108}$ in vivo, as shown both by trapping and $\sigma^{\mathrm{E}}$-induction experiments, even though RseA ${ }^{1-108}$ is a good ClpXP substrate in the absence of SspB in vitro. Furthermore, the use of adaptors can lead to the degradation of a group of proteins, allowing coregulation. The results of our trapping experiments indicate that up-regulation or down-regulation of SspB would be likely to change the efficiency of degradation of a group of substrate proteins in a coordinated manner. In fact, we have observed that overproduction of SspB improves activation of $\sigma^{\mathrm{E}}$ during stress (data not shown). We suspect that additional adaptors remain to be discovered. These proteins, like $\mathrm{SspB}$, will probably also bind a spectrum of substrates, thereby controlling the breadth and efficiency of recognition by their partner AAA+ enzymes.

\section{Materials and methods}

Strains and plasmids

Genes encoding Rse $\mathrm{A}^{1-108}$ and Rse $\mathrm{A}^{1-99}$ were amplified by PCR from E. coli genomic DNA using primers encoding NdeI and BamHI restriction sites. The amplified DNA was cleaved with both restriction enzymes and cloned between the NdeI and BamHI sites of pET3a to generate pET3a-rseA ${ }^{1-108}$ and pET3arse $^{1-99}$. A plasmid expressing RseA-DD ${ }^{1-108}$ was constructed by site-directed mutagenesis of the $r s e A^{1-108}$ gene. The gene encoding $\sigma^{\mathrm{E}}(r p o E)$ was PCR amplified from E. coli chromosomal DNA and cloned into the NdeI and BclI sites of the pT7LysS plasmid (I. Levchenko, unpubl.) to generate pT7LysSrpoE.

The chromosomally encoded $s s p B$ gene was replaced by a FRT-flanked kanamycin resistance cassette following the method of Datsenko and Wanner (2000). The sspB::kan cassette was then transferred into W3110 clpP::cat $\Delta s m p B-1$ cells by $\mathrm{P} 1$ transduction. $\mathrm{Km}^{\mathrm{R}}$ mutants were transformed with pCP20 encoding the Flipase enzyme, and resulting transformants were tested for loss of the kanamycin resistance as described in Datsenko and Wanner (2000). The deletion was confirmed by PCR analysis. A clpA - kan cassette was then introduced by P1 transduction and finally pJF105 (Flynn et al. 2003) encoding the $\mathrm{ClpP}^{\text {trap }}$ was transformed into the strain (JF259). CAG43583 (Walsh et al. 2003) was a gift from Carol Gross (University of California at San Francisco, San Francisco, CA). The sspB · kan and $\operatorname{clpX} \cdot$ kan cassettes were introduced into the strain by $\mathrm{P} 1$ transduction.

\section{Solutions}

Buffer A is $50 \mathrm{mM}$ Tris- $\mathrm{HCl}(\mathrm{pH} 7.0), 50 \mathrm{mM} \mathrm{NaCl}, 0.5 \mathrm{mM}$ DTT, $2 \mathrm{mM}$ EDTA, and $5 \%$ glycerol. GF buffer is $50 \mathrm{mM}$ Tris$\mathrm{HCl}(\mathrm{pH} 7.0), 150 \mathrm{mM} \mathrm{KCl}, 1 \mathrm{mM} \mathrm{DTT}$, and 5\% glycerol. PD buffer is as described (Kim et al. 2000).

\section{Proteins}

ClpX (Levchenko et al. 1997) and ClpP (Kim et al. 2000) were purified as described; SspB was a gift from David Wah (Massachusetts Institute of Technology, Cambridge, MA).

RseA $^{1-108}$ was purified from E. coli ER2556 pLysS/pET3aRseA $^{1-108}$ cells grown in LB broth with $100 \mu \mathrm{g} / \mathrm{mL}$ ampicillin and $30 \mu \mathrm{g} / \mathrm{mL}$ chloramphenicol. Cells were grown at $37^{\circ} \mathrm{C}$ to an 
O.D. ${ }_{600}$ of 0.6 and protein expression was induced with IPTG for $2 \mathrm{~h}$. All purification steps were performed at $4^{\circ} \mathrm{C}$. Cell pellets were resuspended at a concentration of $3 \mathrm{~mL} / \mathrm{g}$ of cells in buffer A plus $6 \mathrm{M}$ of guanidine and Protease Inhibitor Cocktail III (Calbiochem). Following lysis for $1 \mathrm{~h}$, the lysate was centrifuged for $30 \mathrm{~min}$ at $25,000 \times \mathrm{g}$ and the supernatant was dialyzed overnight against $4 \mathrm{~L}$ of buffer A with one buffer change. Insoluble proteins were removed by centrifugation, and ammonium sulfate was added to the supernatant to a final concentration of $30 \%$. After mixing for $20 \mathrm{~min}$, the precipitate containing RseA $^{1-108}$ was collected by centrifugation, resuspended in buffer $\mathrm{A}$, and desalted into the same buffer using a PD-10 desalting column (Amersham Biosciences). This sample was loaded onto a MonoQ HR 5/5 column (Amersham Biosciences) equilibrated in buffer A. The column was washed with 10 -column volumes of buffer $\mathrm{A}$, and the bound protein was eluted with a gradient to $1 \mathrm{M} \mathrm{NaCl}$. The peak, including $\mathrm{RseA}^{1-108}$, was collected, and TFA was added to a final concentration of $0.06 \%$. The sample was applied to a C4 HPLC column equilibrated in $0.06 \%$ TFA to separate full-length RseA ${ }^{1-108}$ from degradation products, and eluted with a gradient to $80 \%$ acetonitrile. RseA ${ }^{1-108}$ was lyophilized, resuspended in buffer $\mathrm{A}$, and dialyzed against the same buffer overnight. RseA ${ }^{1-108}$ concentration was determined by UV absorbance $\left(\varepsilon_{280}=24040 \mathrm{M}^{-1} \mathrm{~cm}^{-1}\right)$.

RseA $^{1-99}$ was purified from E. coli BL-21/pET3a-rseA ${ }^{1-99}$ cells using a similar protocol, except lysis in $50 \mathrm{mM}$ Tris ( $\mathrm{pH} 8.0$ ), $0.15 \mathrm{M} \mathrm{NaCl}, 1 \mathrm{mM}$ EDTA, and $5 \%$ glycerol was performed by French press, and a Superdex 75 column (Amersham Biosciences) was used in place of HPLC as the final purification step. The resulting protein was $>95 \%$ pure as determined by Commassie staining on a SDS-polyacrylamide gel.

The $\sigma^{\mathrm{E}} \cdot$ RseA $^{1-108}$ and $\sigma^{\mathrm{E}} \cdot$ RseA $^{1-108} \mathrm{DD}$ complexes were purified from ER2566 E. coli cells coexpressing either pET3arseA $^{1-108}$ or pET3a-rseA ${ }^{1-108} \mathrm{DD}$ and pT7LysS-rpoE plasmids. The binary complexes were purified on a Sephacryl S-200 gel filtration column (Amersham Biosciences) followed by chromatography on Source15Q (Amersham Biosciences). The RseA fragment purified from the RseA ${ }^{1-108} \cdot \sigma^{\mathrm{E}}$ complex contained a number of C-terminal degradation products. The smallest degradation product $\left(\mathrm{RseA}^{1-89}\right)$ was isolated by a C4 HPLC column as described above, and had a molecular weight of 10,251 Da by electrospray mass spectrometry.

${ }^{35} \mathrm{~S}$-labeled RseA ${ }^{1-108}$ and $\sigma^{\mathrm{E}}$ were purified as a complex from ER2566 E. coli cells coexpressing pET3a-rse $A^{1-108}$ and $p$ T7LysSrpoE. ${ }^{35}$ S-labeling was performed as described (Kim et al. 2000). Cells were lysed in guanidine, the lysate was dialyzed against buffer A, and a $30 \%$ ammonium sulfate cut was performed. The precipitate was resuspended in buffer $\mathrm{A}$, and applied to a protein C4 HPLC column equilibrated in $0.06 \%$ TFA. RseA ${ }^{1-108}$ and $\sigma^{\mathrm{E}}$, were separated by a gradient to $80 \%$ acetonitrile, lyophilized, resuspended in buffer A, and dialyzed against the same buffer overnight. $\sigma^{\mathrm{E}}$ concentration was determined by UV absorbance $\left(\varepsilon_{280}=14650 \mathrm{M}^{-1} \mathrm{~cm}^{-1}\right)$.

Synthetic fluorescein-labeled peptides containing residues 77-108 of RseA (EAQPAPHQWQKMPFWQKVRPWAAQLTQ $M G V A A)$ and of an SsrA tag sequence (NKKGRHGAANDE NYALAA) were synthesized by the Massachusetts Institute of Technology Biopolymers Laboratory and purified by reversephase chromatography on a C4 HPLC column (Vydac).

\section{Protein trapping}

Trapped proteins were isolated from an $s s p B^{+}$strain (JF162; W3110 clpP :: cat clpA::kan $\Delta s m p B-1 /$ pJF105) or an $s s p B^{-}$strain (JF259; see above) and analyzed by two-dimensional gels as described (Flynn et al. 2003). Protein spots from the gel were ex- cised, digested with trypsin, and analyzed by microcapillary reverse-phase HPLC nano-electrospray tandem mass spectrometry using a Finnigan LCQ DECA quadropole ion trap mass spectrometer (Harvard Microchemistry Facility). The two-dimensional spot corresponding to Rse ${ }^{1-108}$ was subjected to in-gel tryptic digestion as described (Rosenfeld et al. 1992; Hellman et al. 1995) and peptides were analyzed by MALDI mass spectrometry at the MIT Biopolymers Facility.

\section{Degradation assays}

$\mathrm{ClpX}_{6}, \mathrm{ClpP}_{14}, \mathrm{ATP}(4 \mathrm{mM})$, and an ATP regeneration system $(50 \mu \mathrm{g} / \mathrm{mL}$ creatine kinase and $2.5 \mathrm{mM}$ creatine phosphate) were mixed in PD buffer and incubated for $2 \mathrm{~min}$ at $30^{\circ} \mathrm{C}$. For gel analysis, RseA ${ }^{1-108}$ or RseA-DD ${ }^{1-108}(2 \mu \mathrm{M})$ was added, and samples were removed at different times and analyzed by SDSPAGE. Bands were visualized using Sypro Orange protein stain (Molecular Probes) on a Fluorimager 595 (Molecular Dynamics). Degradation of ${ }^{35}$ S-labeled proteins were assayed by changes in TCA-soluble radioactivity as described in Burton et al. (2001). When present, the SspB concentration was $0.2 \mu \mathrm{M}$ (monomer equivalents).

\section{Gel filtration of protein complexes}

Gel filtration was performed on a SMART system (Amersham Biosciences) using a Superose 12 column equilibrated in GF buffer at $4^{\circ} \mathrm{C}$. RseA ${ }^{1-108}, \operatorname{RseA}^{1-99}, \operatorname{RseA}^{1-89}$, or the RseA ${ }^{1-108}$. $\sigma^{\mathrm{E}}$ complex $(8 \mu \mathrm{M})$ was incubated with or without $\operatorname{SspB}(8 \mu \mathrm{M}$ monomer equivalents) in GF buffer for $5 \mathrm{~min}$ at $30^{\circ} \mathrm{C}$ prior to chromatography.

\section{Limited trypsin proteolysis}

A total of $5 \mu \mathrm{M}$ RseA ${ }^{1-99}$ was incubated with or without $15 \mu \mathrm{M}$ SspB in $100 \mathrm{mM}$ Tris- $\mathrm{HCl}(\mathrm{pH} 8.9)$ for $5 \mathrm{~min}$ at $30^{\circ} \mathrm{C}$. Trypsin and $\mathrm{RseA}^{1-108}$ were mixed in a 1:93 ratio and samples were taken at different times and analyzed by $18 \%$ Tris-Tricine SDSPAGE. To identify the resulting RseA fragments, a portion of each time point was analyzed by electrospray mass spectrometry and another portion was separated by SDS-PAGE, transferred onto PVDF membrane (Millipore), stained by Ponceau red stain, and subjected to $\mathrm{N}$-terminal sequencing at the Massachusetts Institute of Technology Biopolymers Facility.

\section{Peptide-binding assays}

Binding of SspB to the fluorescein-labeled RseA ${ }^{75-108}$ peptide $(0.1 \mu \mathrm{M})$ was assayed by fluorescence polarization (excitation $467 \mathrm{~nm}$; emission $511 \mathrm{~nm}$ ) at $30^{\circ} \mathrm{C}$ in PD buffer lacking NP-40 using a Fluoromax-2 instrument (ISA, Jobin-Yvon). Binding curves were fit using Kaleidagraph (Synergy Software).

\section{$\beta$-Galactosidase assays}

Overnight cultures were diluted 1:100 to an O.D. ${ }_{600}$ of $\sim 0.025$ and grown at $30^{\circ} \mathrm{C}$ in LB broth with appropriate antibiotics. The cultures were then grown at $30^{\circ} \mathrm{C}$ to an O.D. ${ }_{600}$ of 0.15 and overexpression of the OmpC fusion protein was induced by $0.2 \% \mathrm{~L}-(+)$-arabinose. $\beta$-Galactosidase activities were measured as described (Miller 1972; Mecsas et al. 1993; Ades et al. 1999).

\section{Acknowledgments}

We thank Carol Gross for strains and advice, and Rachna Chaba, Irena Grigorova, and Carol Gross for sharing unpublished re- 
sults. We thank the Harvard Microchemistry Facility for mass spectrometry and members of the Baker and Sauer labs for help, advice, and comments on the manuscript. Supported by NIH grant AI-16892 and HHMI. T.A.B. is an employee of HHMI.

\section{References}

Ades, S.E. 2004. Control of the alternative $\sigma$ factor $\sigma(\mathrm{E})$ in Escherichia coli. Curr. Opin. Microbiol. 7: 157-162.

Ades, S.E., Connolly, L.E., Alba, B.M., and Gross, C.A. 1999. The Escherichia coli $\sigma(\mathrm{E})$-dependent extracytoplasmic stress response is controlled by the regulated proteolysis of an anti- $\sigma$ factor. Genes \& Dev. 13: 2449-2461.

Alba, B.M., Zhong, H.J., Pelayo, J.C., and Gross, C.A. 2001. degS (hhoB) is an essential Escherichia coli gene whose indispensable function is to provide $\sigma$ (E) activity. Mol. Microbiol. 40: $1323-1333$.

Alba, B.M., Leeds, J.A., Onufryk, C., Lu, C.Z., and Gross, C.A. 2002. DegS and YaeL participate sequentially in the cleavage of RseA to activate the $\sigma(\mathrm{E})$-dependent extracytoplasmic stress response. Genes \& Dev. 16: 2156-2168.

Bolon, D.N., Wah, D.A., Hersch, G.L., Baker, T.A., and Sauer, R.T. 2004. Bivalent tethering of SspB to ClpXP is required for efficient substrate delivery: A protein-design study. Mol. Cell 13: 443-449.

Burton, R.E., Siddiqui, S.M., Kim, Y.I., Baker, T.A., and Sauer, R.T. 2001. Effects of protein stability and structure on substrate processing by the ClpXP unfolding and degradation machine. EMBO J. 20: 3092-3100.

Campbell, E.A., Tupy, J.L., Gruber, T.M., Wang, S., Sharp, M.M., Gross, C.A., and Darst, S.A. 2003. Crystal structure of Escherichia coli $\sigma \mathrm{E}$ with the cytoplasmic domain of its antisigma RseA. Mol. Cell 11: 1067-1078.

Dartigalongue, C., Missiakas, D., and Raina, S. 2001. Characterization of the Escherichia coli $\sigma$ E regulon. J. Biol. Chem. 276: $20866-20875$.

Datsenko, K.A. and Wanner, B.L. 2000. One-step inactivation of chromosomal genes in Escherichia coli K-12 using PCR products. Proc. Nat1. Acad. Sci. 97: 6640-6645.

De Las Penas, A., Connolly, L., and Gross, C.A. 1997a. $\sigma$ E is an essential $\sigma$ factor in Escherichia coli. J. Bacteriol. 179: 68626864.

. 1997b. The $\sigma E$-mediated response to extracytoplasmic stress in Escherichia coli is transduced by RseA and RseB, two negative regulators of $\sigma \mathrm{E}$. Mol. Microbiol. 24: 373-385.

Dougan, D.A., Mogk, A., Zeth, K., Turgay, K., and Bukau, B. 2002a. AAA+ proteins and substrate recognition, it all depends on their partner in crime. FEBS Lett. 529: 6-10.

Dougan, D.A., Reid, B.G., Horwich, A.L., and Bukau, B. 2002 b. ClpS, a substrate modulator of the ClpAP machine. Mol. Cell 9: 673-683.

Dougan, D.A., Weber-Ban, E., and Bukau, B. 2003. Targeted delivery of an ssrA-tagged substrate by the adaptor protein $\mathrm{SspB}$ to its cognate AAA+ protein ClpX. Mol. Cell 12: 373-380.

Flynn, J.M., Levchenko, I., Seidel, M., Wickner, S.H., Sauer, R.T., and Baker, T.A. 2001. Overlapping recognition determinants within the ssrA degradation tag allow modulation of proteolysis. Proc. Natl. Acad. Sci. 98: 10584-10589.

Flynn, J.M., Neher, S.B., Kim, Y.I., Sauer, R.T., and Baker, T.A. 2003. Proteomic discovery of cellular substrates of the ClpXP protease reveals five classes of ClpX-recognition signals. Mol. Cell 11: 671-683.

Gonciarz-Swiatek, M., Wawrzynow, A., Um, S.J., Learn, B.A., McMacken, R., Kelley, W.L., Georgopoulos, C., Sliekers, O., and Zylicz, M. 1999. Recognition, targeting, and hydrolysis of the $\lambda \mathrm{O}$ replication protein by the $\mathrm{ClpP} / \mathrm{ClpX}$ protease. I. Biol. Chem. 274: 13999-14005.

Gottesman, S. 2003. Proteolysis in bacterial regulatory circuits. Annu. Rev. Cell. Dev. Biol. 19: 565-587.

Gottesman, S., Roche, E., Zhou, Y., and Sauer, R.T. 1998. The ClpXP and ClpAP proteases degrade proteins with carboxyterminal peptide tails added by the SsrA-tagging system. Genes \& Dev. 12: 1338-1347.

Hellman, U., Wernstedt, C., Gonez, J., and Heldin, C.H. 1995. Improvement of an 'In-Gel' digestion procedure for the micropreparation of internal protein fragments for amino acid sequencing. Anal. Biochem. 224: 451-455.

Kanehara, K., Ito, K., and Akiyama, Y. 2002. YaeL (EcfE) activates the $\sigma^{\mathrm{E}}$ pathway of stress response through a site-2 cleavage of anti-E, RseA. Genes \& Dev. 16: 2147-2155.

Karzai, A.W., Susskind, M.M., and Sauer, R.T. 1999. SmpB, a unique RNA-binding protein essential for the peptide-tagging activity of SsrA (tmRNA). EMBO J. 18: 3793-3799.

Keiler, K.C., Waller, P.R., and Sauer, R.T. 1996. Role of a peptide tagging system in degradation of proteins synthesized from damaged messenger RNA. Science 271: 990-993.

Kim, D.Y. and Kim, K.K. 2003. Crystal structure of ClpX molecular chaperone from Helicobacter pylori. J. Biol. Chem. 278: 50664-50670.

Kim, Y.I., Burton, R.E., Burton, B.M., Sauer, R.T., and Baker, T.A. 2000. Dynamics of substrate denaturation and translocation by the ClpXP degradation machine. Mol. Cell 5: 639648.

Levchenko, I., Smith, C.K., Walsh, N.P., Sauer, R.T., and Baker, T.A. 1997. PDZ-like domains mediate binding specificity in the Clp/Hsp100 family of chaperones and protease regulatory subunits. Cell 91: 939-947.

Levchenko, I., Seidel, M., Sauer, R.T., and Baker, T.A. 2000. A specificity-enhancing factor for the ClpXP degradation machine. Science 289: 2354-2356.

Levchenko, I., Grant, R.A., Wah, D.A., Sauer, R.T., and Baker, T.A. 2003. Structure of a delivery protein for an AAA+ protease in complex with a peptide degradation tag. Mol. Cell 12: $365-372$.

Maurizi, M.R. and Rasulova, F. 2002. Degradation of L-glutamate dehydrogenase from Escherichia coli: Allosteric regulation of enzyme stability. Arch. Biochem. Biophys. 397: 206-216.

Maurizi, M.R., Clark, W.P., Katayama, Y., Rudikoff, S., Pumphrey, J., Bowers, B., and Gottesman, S. 1990. Sequence and structure of Clp P, the proteolytic component of the ATPdependent Clp protease of Escherichia coli. J. Biol. Chem. 265: 12536-12545.

Maurizi, M.R., Thompson, M.W., Singh, S.K., and Kim, S.H. 1994. Endopeptidase Clp: ATP-dependent Clp protease from Escherichia coli. Methods Enzymol. 244: 314-331.

Mecsas, J., Rouviere, P.E., Erickson, J.W., Donohue, T.J., and Gross, C.A. 1993. The activity of $\sigma$ E, an Escherichia coli heat-inducible $\sigma$-factor, is modulated by expression of outer membrane proteins. Genes \& Dev. 7: 2618-2628.

Miller, J.H. 1972. Experiments in molecular genetics. Cold Spring Harbor Press, Cold Spring Harbor, NY.

Missiakas, D., Mayer, M.P., Lemaire, M., Georgopoulos, C., and Raina, S. 1997. Modulation of the Escherichia coli $\sigma \mathrm{E}(\mathrm{RpoE})$ heat-shock transcription-factor activity by the RseA, RseB and RseC proteins. M. Microbiol. 24: 355-371.

Muffler, A., Fischer, D., Altuvia, S., Storz, G., and Hengge-Aronis, R. 1996. The response regulator RssB controls stability of the $\sigma(\mathrm{S})$ subunit of RNA polymerase in Escherichia coli. EMBO T. 15: 1333-1339.

Neher, S.B., Flynn, J.M., Sauer, R.T., and Baker, T.A. 2003a. 
Latent ClpX-recognition signals ensure LexA destruction after DNA damage. Genes \& Dev. 17: 1084-1089.

Neher, S.B., Sauer, R.T., and Baker, T.A. 2003b. Distinct peptide signals in the UmuD and UmuD' subunits of $U m u D / D^{\prime}$ mediate tethering and substrate processing by the ClpXP protease. Proc. Nat1. Acad. Sci. 100: 13219-13224.

Ortega, J., Lee, H.S., Maurizi, M.R., and Steven, A.C. 2004. ClpA and ClpX ATPases bind simultaneously to opposite ends of ClpP peptidase to form active hybrid complexes. J. Struct. Biol. 146: 217-226.

Rezuchova, B., Miticka, H., Homerova, D., Roberts, M., and Kormanec, J. 2003. New members of the Escherichia coli $\sigma \mathrm{E}$ regulon identified by a two-plasmid system. FEMS Microbiol. Lett. 225: 1-7.

Rosenfeld, J., Capdevielle, J., Guillemot, J.C., and Ferrara, P. 1992. In-gel digestion of proteins for internal sequence analysis after one- or two-dimensional gel electrophoresis. Anal. Biochem. 203: 173-179.

Song, H.K. and Eck, M.J. 2003. Structural basis of degradation signal recognition by $\mathrm{SspB}$, a specificity-enhancing factor for the ClpXP proteolytic machine. Mol. Cell 12: 75-86.

Wah, D.A., Levchenko, I., Baker, T.A., and Sauer, R.T. 2002. Characterization of a specificity factor for an AAA+ ATPase: Assembly of SspB dimers with ssrA-tagged proteins and the ClpX hexamer. Chem. Biol. 9: 1237-1245.

Wah, D.A., Levchenko, I., Rieckhof, G.E., Bolon, D.N., Baker, T.A., and Sauer, R.T. 2003. Flexible linkers leash the substrate binding domain of $\mathrm{SspB}$ to a peptide module that stabilizes delivery complexes with the AAA + ClpXP protease. Mol. Cell 12: 355-363.

Walsh, N.P., Alba, B.M., Bose, B., Gross, C.A., and Sauer, R.T. 2003. OMP peptide signals initiate the envelope-stress response by activating DegS protease via relief of inhibition mediated by its PDZ domain. Cell 113: 61-71.

Wang, J., Hartling, J.A., and Flanagan, J.M. 1997. The structure of ClpP at 2.3 A resolution suggests a model for ATP- dependent proteolysis. Cell 91: 447-456.

Wang, L., Elliott, M., and Elliott, T. 1999. Conditional stability of the HemA protein (glutamyl-tRNA reductase) regulates heme biosynthesis in Salmonella typhimurium. J. Bacteriol. 181: 1211-1219.

Weber-Ban, E.U., Reid, B.G., Miranker, A.D., and Horwich, A.L. 1999. Global unfolding of a substrate protein by the Hsp100 chaperone ClpA. Nature 401: 90-93.

Withey, J.H. and Friedman, D.I. 2003. A salvage pathway for protein structures: tmRNA and trans-translation. Annu. Rev. Microbiol. 57: 101-123.

Wojtkowiak, D., Georgopoulos, C., and Zylicz, M. 1993. Isolation and characterization of ClpX, a new ATP-dependent specificity component of the Clp protease of Escherichia coli. J. Biol. Chem. 268: 22609-22617.

Zhou, Y. and Gottesman, S. 1998. Regulation of proteolysis of the stationary-phase $\sigma$ factor RpoS. J. Bacteriol. 180: 11541158 . 


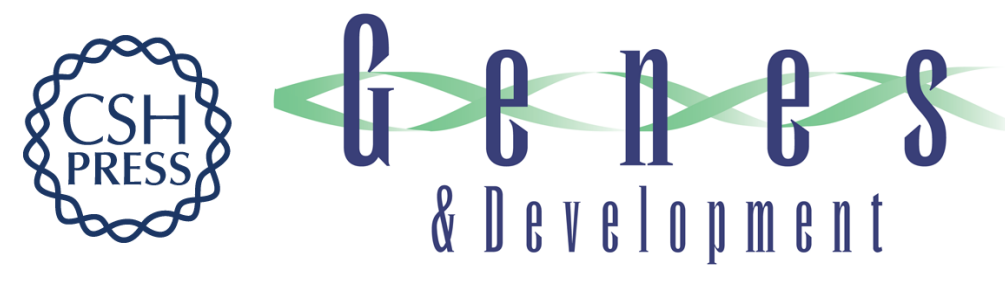

\section{Modulating substrate choice: the SspB adaptor delivers a regulator of the extracytoplasmic-stress response to the AAA+ protease CIpXP for degradation}

Julia M. Flynn, Igor Levchenko, Robert T. Sauer, et al.

Genes Dev. 2004, 18:

Access the most recent version at doi:10.1101/gad.1240104

References This article cites 48 articles, 21 of which can be accessed free at:

http://genesdev.cshlp.org/content/18/18/2292.full.html\#ref-list-1

License

Email Alerting

Receive free email alerts when new articles cite this article - sign up in the box at the top

Service

right corner of the article or click here.

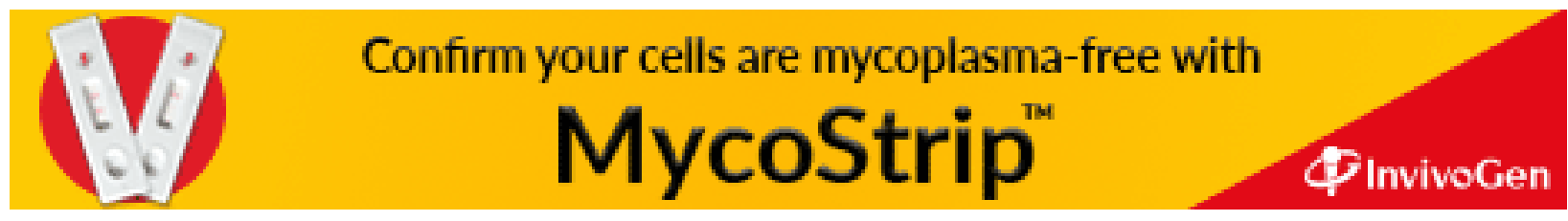

